\title{
Cases report of unicentric Castleman's disease: revisit of radiotherapy role
}

\author{
0 Kyu Noh, MD1, Sang-Wook Lee, MD², Jae Whan Lee, MD³ Sang Yoon Kim, MD, Chung Soo Kim, MD, \\ Eun Kyung Choi, MD², Jong Hoon Kim, MD², Seung Do Ahn, MD² \\ 'Department of Radiation Oncology, Ajou University School of Medicine, Suwon; \\ Departments of ${ }^{2}$ Radiation Oncology, ${ }^{3} \mathrm{Hematology},{ }_{1}^{4}$ Otolaryngology, and ${ }^{5}$ Urology, Asan Medical Center, \\ University of Ulsan College of Medicine, Seoul, Korea
}

Castleman's disease or angiofollicular lymph node hyperplasia is a rare lymphoproliferative disorder. Complete surgical resection was recommended in unicentric Castleman's disease. Radiotherapy was considered alternative therapeutic option. However, there have been consistent favorable responses to radiotherapy. We also experienced two cases of uncentric Castleman's disease salvaged successfully with radiotherapy. This paper described these cases and reviewed the literature about Castleman's disease treated with radiotherapy. Reviewed cases showed that radiotherapy is a successful treatment option in unicentric Castleman's disease. Furthermore, our report confirms the radiotherapy role in uncentric Castleman's disease.

Keywords: Castleman's disease, Hyaline-vascular, Radiotherapy

\section{Introduction}

Castleman's disease (CD) or angiofollicular lymph node hyperplasia (AFLNH) was first described as a case of peculiar form of massive lymph node hyperplasia characterized by germinal center formation and marked capillary proliferation in 1954, and later defined by Castleman et al. [1] in 1956. Pathophysiology of this rare lymphoproliferative disorder is still unknown. However, association with human herpesvirus 8 (HHV-8) infection is reported in disseminated cases with human immunodeficiency virus (HIV) patients [2]. CD has two distinct pathologic types, which called hyaline-vascular type and plasma cell type [3]. Clinically, CD is classified as unicentric (localized) or multicentric CD according to the extent of disease. Majority of unicentric CD is hyaline-vascular type and presents with localized mass effect or free of symptom. Clinical course of unicentric $C D$ is benign in most cases. On the other hand, multicentric $C D$ is mostly plasma cell type and has a poor clinical course than unicentric CD [4]. Initial presentations of multicentric $C D$ are disseminated lymphadenopathy, systemic symptom (fever, fatigue, sweating, and weight loss), hepatosplenomegaly and sometimes associated with autoimmune disease.

There is no consensus about treatment strategy in this rare and poorly understood disease. Generally, surgical resection or radiotherapy is performed in unicentric $C D$ and systemic therapy such as steroid, chemotherapy is used in multicentric type. In unicentric $C D$, complete surgical resection is considered standard therapy with excellent results. Radiotherapy has been thought as an alternative treatment

Received 1 November 2012, Revised 5 December 2012, Accepted 13 December 2012.

Correspondence: Sang-Wook Lee, MD, Department of Radiation Oncology, Asan Medical Center, University of Ulsan College of Medicine, 88 Olympic-ro 43-gil, Songpa-gu, Seoul 138-736, Korea. Tel: +82-2-3010-4435, Fax: +82-2-486-7258, E-mail: Isw@amc.seoul.kr

(c) This is an Open Access article distributed under the terms of the Creative Commons Attribution Non-Commercial License (http://creativecommons.org/ licenses/by-nc/3.0/) which permits unrestricted non-commercial use, distribution, and reproduction in any medium, provided the original work is properly cited.

www.e-roj.org 
option with curative potential in some cases. Because of unfavorable reports about radiotherapy by Keller et al. [3] in 1972, the role of radiotherapy has been underestimated. However, consistent favorable reports suggested that unicentric $C D$ can be successfully treated with radiotherapy. We also experienced two cases of unicentric CD salvaged successfully with radiotherapy. In this paper, we describe the detail of cases and reviewed the role of radiotherapy in the literature.

\section{Case Reports}

\section{Case 1}

A twenty-year-old female referred with the impression of inflammatory pseudotumor on left supraclavicular area. She visited provincial hospital with 3 months history of hoarseness and left neck mass. Laboratory test such as complete blood cell count $(\mathrm{CBC})$ and chemistry did not show any abnormality.
At that time, left true vocal cord palsy was found on laryngoscopic examination. On the computed tomography (CT) scan, there was a heterogenous enhanced and carotid artery encasing huge mass with multiple enlarged lymph nodes from left level IV Iymph node station to supraclavicular area (Fig. 1A). Incisional biopsy was done and pathologic examination showed infiltrations of heterogenous inflammatory cells with fibroblastic proliferations. Diagnosis was not definite and referred for the further work-up and appropriate treatment. For the definite diagnosis, incisional biopsy was performed once more. On the pathologic review, small lymphocytes, plasma cells, and eosinophilic infiltrations were seen. Inflammatory pseudotumor, Kimura disease, Hodgkin's disease, and CD were discussed as possible diagnosis, but were not conclusive. For more precise pathologic examination, excisional biopsy was done under general anesthesia. Mass was not fully excised because of carotid artery encasement (Fig. 1B). However, vocal
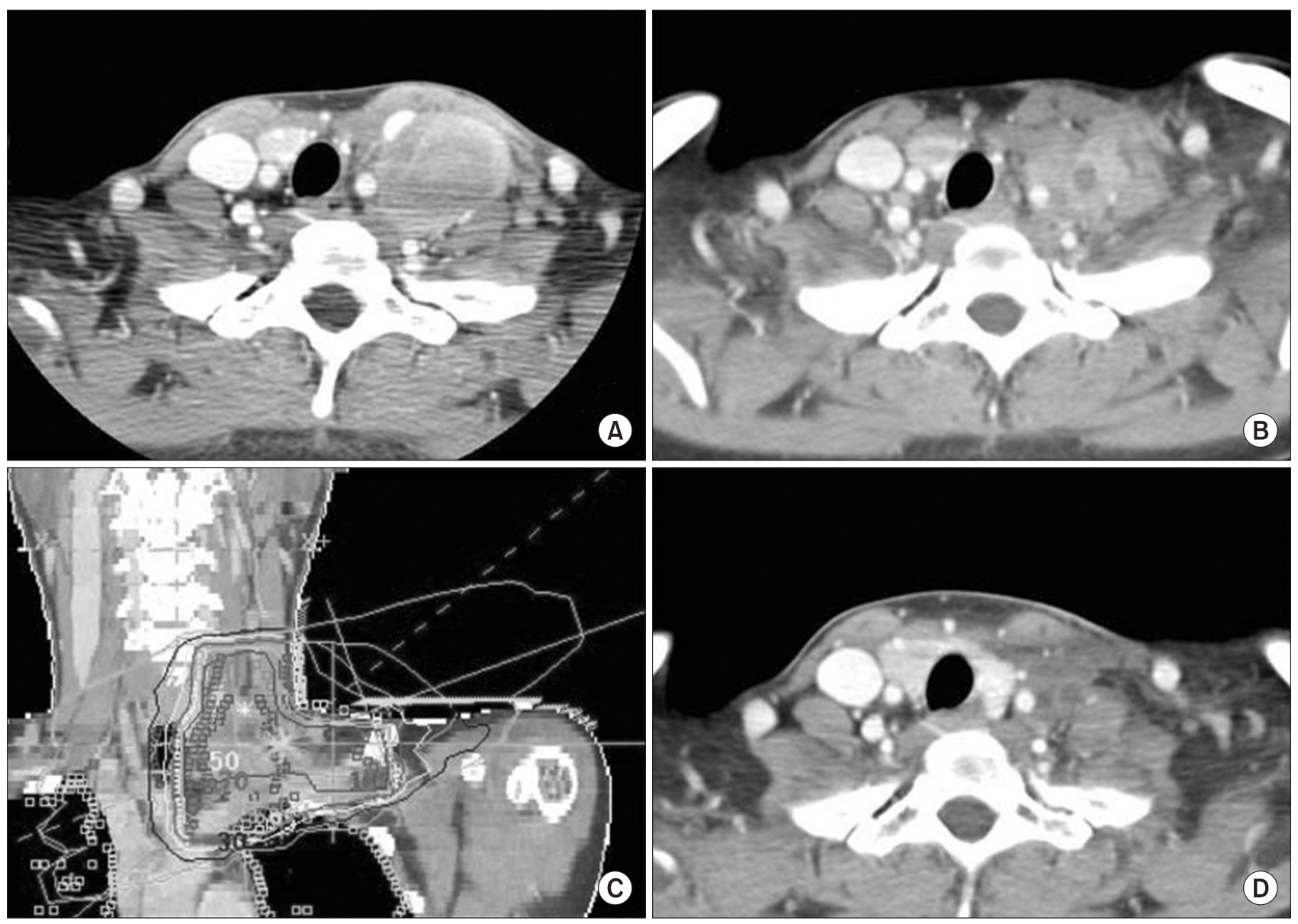

Fig. 1. Case 1. (A) Initial neck computed tomography scan, (B) postexcisional state, (C) radiation therapy planning, and (D) partial response to radiation therapy. 
cord palsy was recovered after excisional biopsy (Fig. 1B). In immunohistochemical studies, CD3 and CD20 were negative and CD21 was positive in follicular dendritic cells. Kappa and lambda in situ hybridization (ISH) revealed polyclonality and the Epstein-Barr virus (EBV) ISH was negative. Ki-67 labeling index was not increased. Patient was diagnosed as hyalinevascular type $C D$ on excisional specimen and consulted for postoperative radiotherapy to residual mass. Patient was treated with 3-dimensional conformal radiotherapy on 2 months after surgery. The target volume included tumor bed with generous margin. Radiation dose of 36 Gy/20 fractions was delivered for 30 days and radiotherapy was completed without treatment related complication except mild radiation esophagitis (Fig. 1C). Three months later after radiotherapy, CT imaging showed a partial response of residual mass (Fig. 1D). On follow-up CT scan at 14 months after radiotherapy, there was additional decrease in mass size.

\section{Case 2}

The patient was a 51-year-old woman who had huge mass in left perirenal space. She took a medical examination because of easy fatigability and headache. On CT scan, there was about $13 \mathrm{~cm}$ sized mass originated from left perirenal space with diffusely conglomerated multiple lymphadenopathy in left para-aortic space (Fig. 2A). Chest X-ray, bone scan, laboratory test such as CBC and chemistry did not show any abnormal findings. On ultrasonograpy, perirenal mass had few vascularity in it and seated in hilum of left kidney. In addition, about $2 \mathrm{~cm}$ sized multiple lymphadenopathy were seen around abdominal aorta. Ultrasonography-guided biopsy of perirenal mass revealed polymorphorous lymphoid proliferation with stromal hyalinization. Proliferated cells consisted with small B
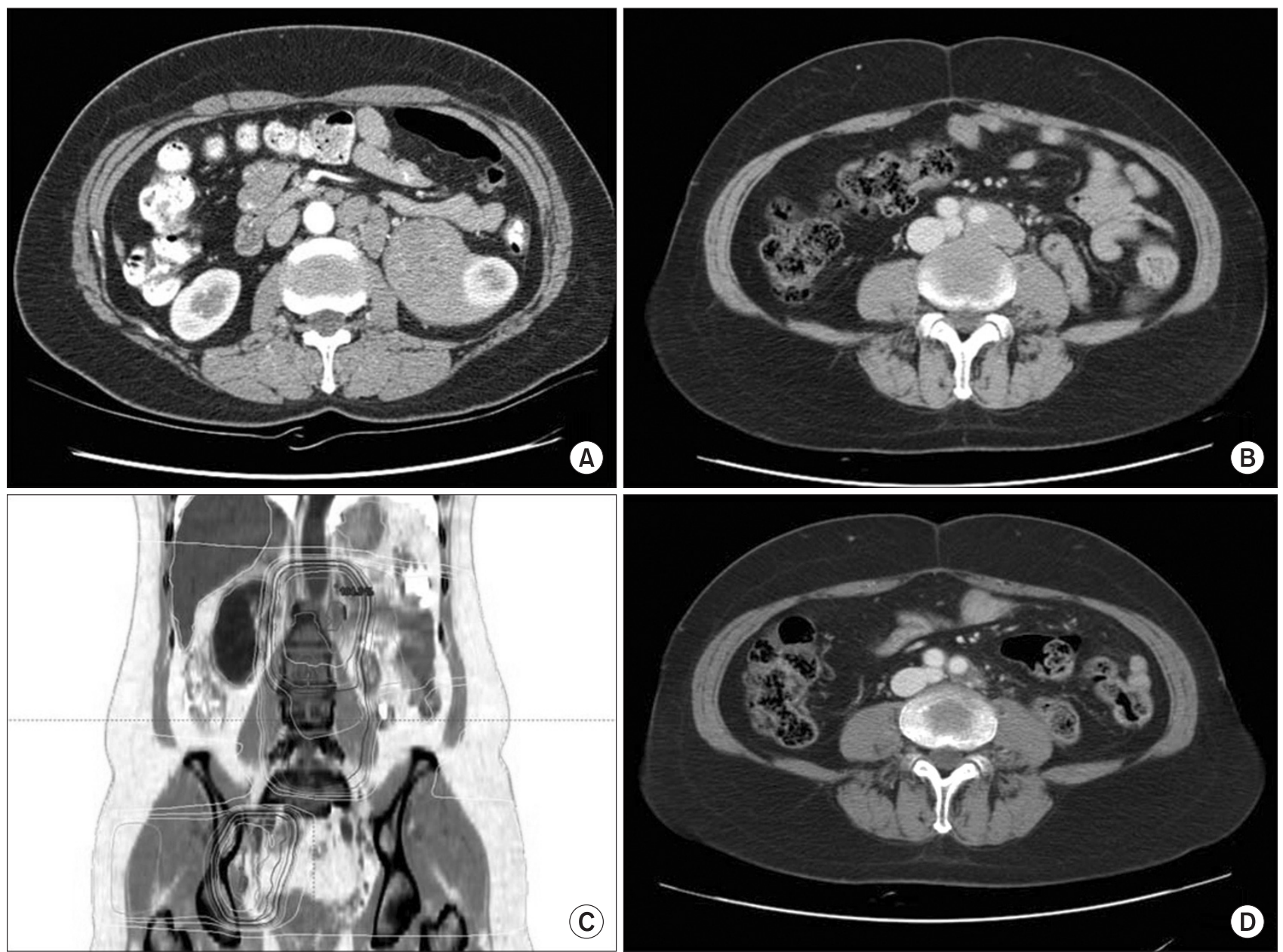

Fig. 2. Case 2. (A) Initial abdomen computed tomography scan, (B) recurrent para-aortic lymphadenopathy, (C) radiation therapy planning, and (D) partial response to radiation therapy. 
and T lymphocytes, and mature plasma cells. Polymorphorous infiltration and stromal hyalinization suggested a benign lymphoproliferative lesion such as CD. Mass excision including left kidney and enlarged para-aortic lymph nodes was done without residual mass. Pathologic examination showed $C D$, hyaline-vascular type involving perinephric and paraaortic lymph nodes. By Immunohistochemical staining, the lymphoid cells were polymorphorous and polyclonal and there was occasional EBV-ISN positive cells. Polymerase chain reaction for HHV-8 and $B$ cell immunoglobulin heavy chain gene rearrangement were negative. After surgical resection, the patients received regular follow-up with abdominopelvic CT scan. After disease-free state during 52 months, multiple enlarged lymph nodes were found in follow-up CT scan (Fig. 2B). Enlarged lymph nodes were seen around left para-aortic and common iliac artery bifurcation. Fludeoxyglucose (18F)positron emission tomography (FDG-PET) showed linear hypermetabolism (maxSUV = 3.0) along left side para-aortic and focal hypermetabolism (maxSUV $=1.9)$ in right iliac area. She was consulted for salvage radiotherapy to recurrent lesions. Three-dimensional conformal radiotherapy was performed. Left pelvic lesion was far from para-aortic lymph nodes. Then, two separate radiotherapy plans were needed (Fig. $2 \mathrm{C}$ ). Radiation dose was $40 \mathrm{~Gy} / 20$ fractions with daily fraction size of 2 Gy. At 1 month after radiotherapy, CT scan was done and showed partial response with decrease of lymph node size less than $1 \mathrm{~cm}$ (Fig. 2D). Twelve months after radiotherapy the patient had no evidence of disease on follow-up CT scan.

\section{Discussion}

CD presents with single or multiple lymph node hyperplasia with benign course in most cases. Histopathology of $C D$ is unknown, but seems to be associated with chronic low-grade inflammation, immunodeficiency state, and autoimmunity. In some cases, EBV, Toxoplasma, Mycobacterium tuberculosis is considered as etiologic factors. Especially, HHV-8 is proved its significant role in development of plasmablastic form of multicentric CD. The diagnosis must consider after more common other cause of lymphadenopathy have been searched. Typical histologic findings are small hyaline-vascular follicles with interfollicular capillaries (hyaline-vascular type), and large follicles with intervening sheets of plasma cells (plasma cell type) [3]. There is mixed type CD with both characteristics of hyaline-vascular type and plasma cell type. In most cases more than 90\%, CD involves single or localized lymph node (unicentric or localized CD). Contrary, involving multiple lymph nodes in about $10 \%$ is designated with multicentric CD [5] Unicentric hyaline-vascular disease is seen in 70\% of total cases of CD, and 90\% of unicentric disease. Most of them present with large node in mediastinum, neck, abdomen, axilla, and so on. Unicentric hyaline-vascular type CD is asymptomatic in almost cases. Sometimes, symptoms by mass effect or constitutional symptoms may be present. Incisional biopsy is not sufficient for definite diagnosis in some cases. So, excisional biopsy may be required for definite diagnosis by review of entire lymph node architecture. Unicentric plasma cell type CD is seen in 10\% of localized disease and often accompany with constitutional symptom.

The treatment of unicentric CD regardless of cell type is surgical resection and complete resection is curative in most cases $[4,6]$. After the surgical resection, systemic symptoms subside within 30-60 days. When the surgical resection is impossible, partial resection or observation will be useful. Radiation therapy can be considered as alternative treatment. Keller et al. [3] reported 6 patients with incomplete resection. In spite of no further treatment, only one patient showed disease progression in these patients. Furthermore, radiation therapy of 1,800-4,500 cGy to hyaline-vascular type CD showed no response. By Keller's review [3], radiotherapy in CD showed unfavorable impression to physicians. However in Keller's four cases, there was no clinical description of each case about clinical findings and treatment details such as radiation technique. When Keller's review was published in 1972, radiation technique was simple and could be inappropriate to treat the deep seated lesion. So, Keller's clinical outcome after radiotherapy is hard to accept as it was reported. When we reviewed the literature about $C D$, results of radiotherapy to $C D$ was comparable to surgical outcome. There were 27 cases treated with radiotherapy including our report (Table 1) [4-15]. Complete response showed in 12 patients, partial response in 7, no response in 7, and progression in 1, respectively. Overall response rate is about 70\% (19/27) and almost responding patients sustained stable or remission status. In only 3 patients, there were no responses after radiotherapy except Keller's four patients [3]. Two of them followed up without symptoms until 12 and 50 months. And the last patient, in Gaba's report [5], she had multicentric CD with huge mass, ascites, and organomegaly. Despite extensive extent of disease, she had followed up to 2 years with stable disease. There was a disease progression in only one patient [15]. In this case, a 71-year-old man had a diffusely infiltrating mediastinal mass 


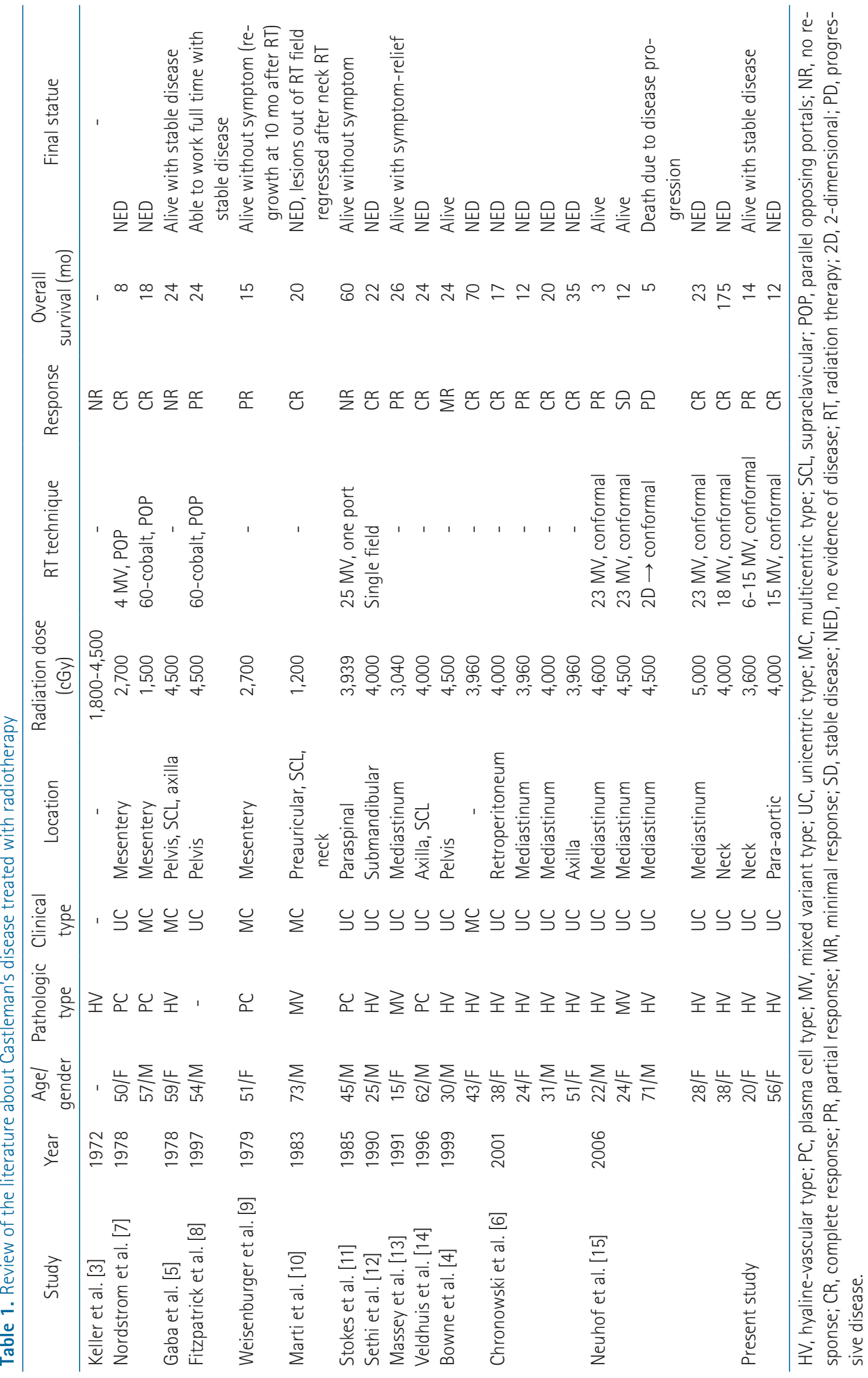


with pleural and pericardial effusion and history of spinal radiotherapy due to bone metastasis from prostate cancer. He died of progressive CD at three months after radiotherapy despite initial partial response. Although this case showed that radiotherapy had a limited role in diffusely infiltrating $C D$, radiotherapy to unicentric $C D$ showed good control rate.

Our two cases show again that radiotherapy is one of the most effective treatments to unicentric CD just as surgery do. As the techniques of radiotherapy developed, it is possible to deliver high-dose radiation to target tumor with minimal complication rate. In modern radiation technology, use of new radiotherapy techniques such as three-dimensional conformal radiation therapy or intensity modulated radiation therapy has been generalized. Radiation dose around 4,000 cGy, that is sufficient dose to control CD, can be delivered safely and easily without complication. In the review of literature, all cases after using high-energy photon were controlled more than stable disease except diffusely infiltrating mediastinal tumor of Neuhof's report [15]. Radiotherapy is less invasive and performed on the basis of out-patient. On the other hand, surgical resection is more invasive, sometimes it results in irreversible surgical complication. It is not always possible in the case of tumor close to critical organ. Moreover, unicentric $C D$ is not a malignant disease definitely, but close to benign with good prognosis. Therefore radiation therapy can be a more favorable treatment to unicentric $C D$ than invasive surgical resection.

Our first case was unicentric hyaline-vascular type and showed a typical clinical course. There were no symptoms except vocal cord palsy due to mass effect. Definitive diagnosis was confirmed by excision after repetitive incisional biopsy. Surgical excision was not complete because of vessel encasement. However, mass reduction by partial excision resulted in improvement of vocal cord palsy. Radiation dose of 36 Gy was used and this was sufficient to attain good response. Second case could be considered as multicentric $\mathrm{CD}$ because of multiple lymph nodes involvement. However, clinical course is similar to unicentric CD. In fact, there is no clear criterion between unicentric and multicentric diseases. Number of involved lymph nodes cannot be absolute criteria. In first description by Castleman et al. [1], single lymph node was involved in most cases. But, adjacent small lymph nodes were found in a few cases. Gaba et al. [5] described first multiple lymph node involvement of hyalinevascular type $C D$. Involved lymph nodes were located in retroperitoneal, axillary area, and splenomegaly, polyclonal gammopathy, neuropathy were accompanied. Later, Frizzera et al. [16] reported 15 cases of systemic lymphoproliferative disorder with features of $C D$. In this report, multicentric disease were characterized by 1) plasma cell type, 2) multiple lymphadenopathy (preferentially peripheral nodal groups), 3) multisystemic involvement (such as constitutional symptom, splenomegaly, hypergammaglobulinemia, increased erythrocyte sedimentation rate, thrombocytopenia) and 4) idiopathic nature. Multicentric disease was classified by combination of these characteristics. Especially, multicentric CD showed a devastating course in conjunction with neuropathy and sometimes associated with malignancy such as lymphoma. In a word, multicentric disease is systemic disorder with poor clinical course. So, our second case was close to unicentric or localized disease with benign clinical course. The main pathologic type of multicentric CD is plasma cell type with poor prognosis, but hyaline-vascular type show good prognosis expecting long term survival [4]. In general, systemic therapy such as steroid, systemic chemotherapy is used in treatment of multicentric CD. In the setting of failure of systemic chemotherapy, high-dose of chemotherapy followed by autologous bone marrow transplantation can be considered [17]. Immune modulators such as interferon alpha [18], all-trans retinoic acid [19], anti-interleukin-6 monoclonal antibody [20] and antiviral agents can be tried also. Radiation therapy can be considered as palliative aim in multicentric CD. However, there were several reports that showed considerable response of radiation therapy in multicentric CD [6].

Role of radiation therapy has been underestimated or misunderstood because of early reports with poor response to radiotherapy. However, radiotherapy can be a definitive treatment modality of unicentric CD with good control rate and low complication in the review of literature including this case report.

\section{Conflicts of Interest}

No potential conflict of interest relevant to this article was reported.

\section{References}

1. Castleman B, Iverson L, Menendez VP. Localized mediastinal Iymphnode hyperplasia resembling thymoma. Cancer 1956:9:822-30.

2. Dupin $N$, Diss TL, Kellam P, et al. HHV-8 is associated with a 
plasmablastic variant of Castleman disease that is linked to HHV8-positive plasmablastic lymphoma. Blood 2000;95:1406-12.

3. Keller AR, Hochholzer L, Castleman B. Hyaline-vascular and plasma-cell types of giant lymph node hyperplasia of the mediastinum and other locations. Cancer 1972;29:670-83.

4. Bowne WB, Lewis JJ, Filippa DA, et al. The management of unicentric and multicentric Castleman's disease: a report of 16 cases and a review of the literature. Cancer 1999;85:70617.

5. Gaba AR, Stein RS, Sweet DL, Variakojis D. Multicentric giant Iymph node hyperplasia. Am J Clin Pathol 1978;69:86-90.

6. Chronowski GM, Ha CS, Wilder RB, Cabanillas F, Manning J, Cox JD. Treatment of unicentric and multicentric Castleman disease and the role of radiotherapy. Cancer 2001;92:670-6.

7. Nordstrom DG, Tewfik HH, Latourette HB. Giant lymph node hyperplasia: a review of literature and report of two cases of plasma cell variant responding to radiation therapy. Int J Radiat Oncol Biol Phys 1978;4:1045-8.

8. Fitzpatrick PJ, Brown TC. Angiofollicular lymph node hyperplasia. Can Med Assoc J 1968;99:1259-62.

9. Weisenburger DD, DeGowin RL, Gibson P, Armitage JO. Remission of giant lymph node hyperplasia with anemia after radiotherapy. Cancer 1979;44:457-62.

10. Marti S, Pahissa A, Guardia J, Moragas A, Bacardi R. Multicentric giant follicular lymph node hyperplasia: favorable response to radiotherapy. Cancer 1983;51:808-10.

11. Stokes SH, Griffith RC, Thomas PR. Angiofollicular lymph node hyperplasia (Castleman's disease) associated with vertebral destruction. Cancer 1985;56:876-9.

12. Sethi $T$, Joshi $K$, Sharma SC, Gupta BD. Radiation therapy in the management of giant lymph node hyperplasia. Br J Radiol
1990;63:648-50.

13. Massey GV, Kornstein MJ, Wahl D, Huang XL, McCrady CW, Carchman RA. Angiofollicular lymph node hyperplasia (Castleman's disease) in an adolescent female: clinical and immunologic findings. Cancer 1991;68:1365-72.

14. Veldhuis GJ, Van Der Leest AH, De Wolf JT, De Vries EG, Vellenga $E$. A case of localized Castleman's disease with systemic involvement: treatment and pathogenetic aspects. Ann Hematol 1996;73:47-50.

15. Neuhof D, Debus J. Outcome and late complications of radiotherapy in patients with unicentric Castleman disease. Acta Oncol 2006;45:1126-31.

16. Frizzera G, Peterson BA, Bayrd ED, Goldman A. A systemic lymphoproliferative disorder with morphologic features of Castleman's disease: clinical findings and clinicopathologic correlations in 15 patients. J Clin Oncol 1985;3:1202-16.

17. Repetto $L$, Jaiprakash MP, Selby PJ, Gusterson BA, Williams HJ, McElwain TJ. Aggressive angiofollicular lymph node hyperplasia (Castleman's disease) treated with high dose melphalan and autologous bone marrow transplantation. Hematol Oncol 1986;4:213-7.

18. Andres $E_{1}$ Maloisel F. Interferon-alpha as first-line therapy for treatment of multicentric Castleman's disease. Ann Oncol 2000;11:1613-4.

19. Rieu P, Droz D, Gessain A, Grunfeld JP, Hermine O. Retinoic acid for treatment of multicentric Castleman's disease. Lancet 1999;354:1262-3.

20. Nishimoto $N$, Sasai $M$, Shima $Y$, et al. Improvement in Castleman's disease by humanized anti-interleukin-6 receptor antibody therapy. Blood 2000;95:56-61. 